\title{
Usage criminel de produits psychoactifs : analyse d'une série parisienne
}

\section{Criminal use of psycho-active drugs : analysis of a cohort in Paris}

Frank QUESTEL ${ }^{*(1,2)}$, Georges LAGIER ${ }^{(2)}$, Dominique FOMPEYDIE ${ }^{(3)}$, Samira DJEZZAR ${ }^{(4)}$, Sylvain DALLY ${ }^{(4)}$, David ELKHARRAT ${ }^{(5)}$, Odile DIAMANT-BERGER ${ }^{(1)}$

(1) Urgences Médico-Judiciaires, Hôpital Hôtel-Dieu - PARIS (2) Centre Anti-Poison et de Pharmacovigilance, Hôpital Fernand Widal - PARIS (3) Laboratoire de Toxicologie, Hôpital Fernand Widal - PARIS (4) Centre d'Évaluation et d'Information sur la Pharmacodépendance, Hôpital Fernand Widal - PARIS (5) Service d'Accueil et de traitement des Urgences (SAU), Hôpital Lariboisière - PARIS

* Auteur à qui adresser la correspondance : Frank QUESTEL, Urgences Médico-Judiciaires, Hôpital Hôtel-Dieu, 1, place du Parvis Notre Dame - 75004 PARIS - Tél : 0142348229

(Reçu le 2 octobre 2002 ; accepté le 21 octobre 2002)

\section{$R \hat{E} S U M E ́$}

L'administration de psychotrope à l'insu d'une victime est un mode d'agression méconnu dont la fréquence est mal évaluée. Une étude impliquant des services cliniques et d'information toxicologique Parisiens a permis le recensement de 137 observations entre 1993 et 2001 . Ont été étudiés les circonstances de l'agression, la symptomatologie retrouvée chez la victime, les résultats des analyses toxicologiques, l'imputabilité du psychotrope identifié. Les infractions sont majoritairement des vols et des agressions sexuelles. Les produits psycho-actifs ont été incorporés dans des boissons, alcoolisées ou non, plus rarement dans des aliments. Les produits les plus souvent utilisés sont des benzodiazépines ou analogues ( $87 \%)$; toutes sont représentées notamment bromazépam, lorazépam, funitrazépam et zolpidem. Les autres. substances incriminées sont beaucoup plus rarement des antihistaminiques sédatifs, des neuroleptiques et du GHB.

\section{SUMMARY}

Administration of psychoactive drugs without the victim's knowledge is a mode of agression that is still poorly identified and estimated. A study implicating clinical units and toxicological information centers has allowed the collection of 137 cases beetween 1993 and 2001. Circumstances of the agression, victim's symptomatology, toxicological results, causal relationship of the psychotropic drug identified were studied. The offences were predominantly thefts and sexual agressions. Psychoactive drugs were introduced into alcoholic or non alcoholic drinks, or more rarely into food. The substances most frequently used were benzodiazepines and analogues ( $87 \%)$; all members of this family were represented, especially bromazepam, lorazepam, funitrazépam and zolpidem. The other substances incriminated more rarely were sedative antihistamines, neuroleptics and $G H B$. Teses substances were associated with alcohol in one-third 
L'association à l'alcool est retrouvée 1 fois sur 3, au cannabis 1 fois sur 5. Les victimes doivent être médicalisées rapidement afin de favoriser des prélèvements à visée toxicologique précoces. Ceux-ci doivent bénéficier de techniques analytiques chromatographiques en complément des techniques immunochimiques de sensibilité et de spécificité insuffisantes. Le relais médico-légal doit être favorisé en sollicitant les structures médico-judiciaires existantes ; le recensement des observations doit se poursuivre au niveau national afin de quantifier le phénomène et de surveiller l'apparition de nouveaux produits.

\section{MOTS-CLÉS}

Soumission, victimologie, toxicologie, psychotropes, chromatographie.

\section{Introduction}

L'administration de produits psycho-actifs à l'insu d'une victime à des fins délictueuses (vols) ou criminelles (agressions sexuelles) est un mode d'agression méconnu. Ce phénomène, encore appelé «soumission chimique», est en augmentation comme l'attestent de récents témoignages, publications et communications scientifiques (1-5). Les produits incriminés sont le plus souvent des médicaments hypnotiques ou anxiolytiques de type benzodiazépines aux propriétés sédatives et amnésiantes ; d'autres médicaments ou substances chimiques dont les stupéfiants peuvent être en cause (2),(4),(6).

Le recensement d'observations est nécessaire à l'évaluation du phénomène et à l'élaboration de recommandations relatives à la prise en charge des victimes; il a été facilité à Paris par la mise en place d'un réseau regroupant services cliniques, centre anti-poison (CAP), centre d'évaluation et d'informations sur la pharmacodépendance (CEIP) et centre régional de pharmacovigilance (CRPV). La majorité des analyses toxicologiques disponibles ont été réalisées par le laboratoire de toxicologie de l'hôpital Fernand Widal (Paris).

Cent trente sept (137) observations avec identification du produit psycho-actif ont été répertoriées entre 1993 et 2001 .

\section{Matériel et méthodes}

\section{Recensement des observations}

Plusieurs services cliniques et d'information toxicologique Parisiens ont collaboré au recensement des observations : le Service d'Accueil et de traitement des Urgences (SAU) de l'hôpital Lariboisière et les Urgences médico-judiciaires de l'Hôtel-Dieu (service expert près la cour d'appel de Paris qui reçoit les victimes après dépôt de plainte, sur réquisition judiciaire), le Centre anti-poison et de pharmacovigilance de l'hô- of cases, and with cannabis in 1 out of 5 cases. Victims must be rapidly medicalized to allow early collection of samples for toxicological studies. They must benefited from analysis with chromatographic techniques in complement of immunochemical techniques which present insufficient sensitivity and specificity. Medicolegal support must be encouraged by the solicitation of the existing legal emergency departments, The collection of cases must be pursued to quantified the phenomenon and to supervised the appearance of new sub. stances.

\section{KEY-WORD}

Submission, victimology, toxicology, psychotropes, chromatography.

pital Fernand Widal (qui reçoit des appels téléphoniques de particuliers et de professionnels de santé) et le Centre d'évaluation et d'information sur les pharmacodépendances de l'hôpital Fernand Widal (qui reçoit des appels téléphoniques de professionnels de santé et des services de police). Le Centre régional de pharmacovigilance de l'hôpital Fernand Widal a collecté les observations et coordonné l'étude. (Dans le même temps, le Centre régional de l'hôpital Fernand Widal a coordonné cette enquête au plan national avec la collaboration des réseaux des CRPV ( 31 centres), des CEIP ( 6 centres), des CAP ( 10 centres)).

Ont été retenues les observations de victimes d'agression sexuelle, de vol ou de violences volontaires présentant une symptomatologie évocatrice d'une soumission chimique (amnésie, désorientation, troubles de la vigilance), et dans lesquelles a été identifié au moins un produit psycho-actif susceptible d'avoir été administré à la victime à son insu. Les observations sans identification de produit psycho-actif ou avec seulement mise en évidence d'un produit psycho-actif ingéré volontairement (alcool, cannabis), n'ont pas été prises en compte.

\section{Période d'étude et origine des cas}

Le recensement des observations a débuté en Octobre 1998 et s'est achevé en Mars 2001 ; il a été rétrospectif pour les cas antérieurs à 1998 et prospectif pour les cas survenus depuis 1998. La période étudiée est comprise entre 1993 et 2001.

Cent trente sept (137) observations avec identification du produit psycho-actif ont été répertoriées : 90 aux Urgences médico-judiciaires de l'Hôtel-Dieu (Paris), 16 dans le Service d'Accueil et de traitement des Urgences de 1'hôpital Lariboisière (Paris), 24 sont des rapports d'expertises judiciaires concernant des affaires jugées, 7 proviennent d'appels téléphoniques ( 6 de particuliers et 1 d'un commissariat). Toutes les observations sont localisées en région Parisienne (Fig1)., 


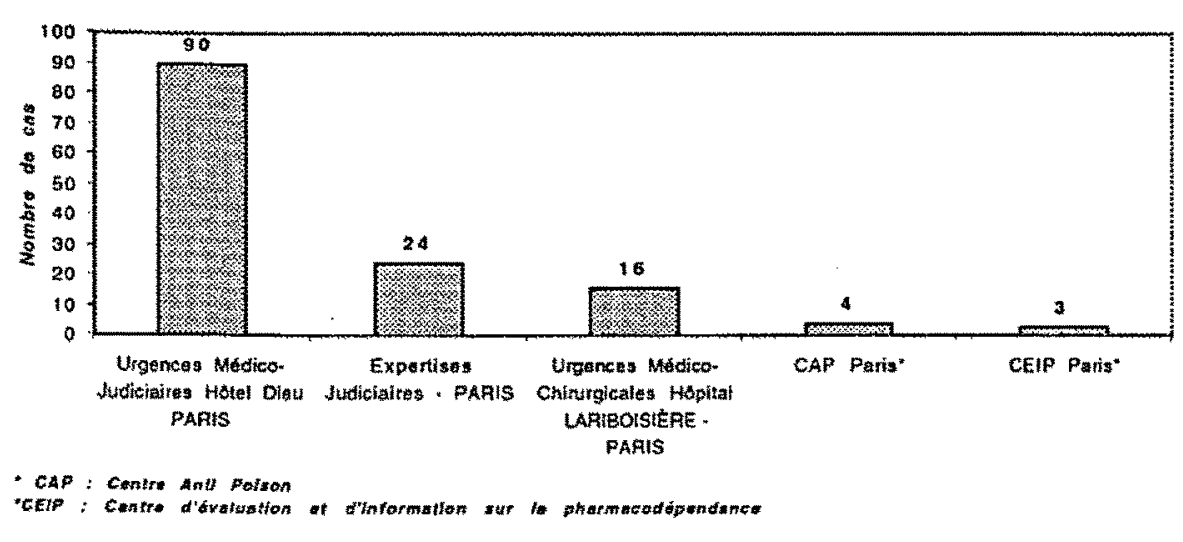

Figure 1 : Origine des cas.

\section{Critères analysés}

Les critères suivants ont été étudiés :

. La date des faits présumés.

. Le profil de la victime : sexe, âge, antécédents médicaux, traitement en cours.

. Le profil du ou des agresseurs.

. La nature de l'agression.

- La nature du produit (boisson; aliment, cachet, contenu d'une seringue...) susceptible de contenir la substance psycho-active.

. Les résultats des analyses toxicologiques.

. L'imputabilité de la substance psycho-active identifiée.

- L'imputabilité a été considérée comme forte dans les circonstances suivantes:

$\Rightarrow$ Substance psycho-active non consommée volontairement retrouvée avec des délais compatibles dans les liquides biologiques, soit de façon isolée, soit associée à un autre psychotrope pris volontairement (alcool, stupéfiants, traitement psychotrope habituel).

$\Rightarrow$ Aveux de l'agresseur.

$\Rightarrow$ Produit psycho-actif administré sous la contrainte, retrouvé dans les liquides biologiques.

- L'imputabilité a été considérée comme douteuse lorsque les circonstances de l'agression sont floues (allégations discordantes, constatations peu compatibles, alcoolisation massive) et que les analyses toxicologiques sont non contributives (mise en évidence de façon isolée de substances psycho-actives volontairement consommées par la victime : alcool, stupéfiants, traitement en cours).

\section{Résultats}

Cent trente sept (137) dossiers avec identification de produit psycho-actif ont été répertoriés.

\section{Répartition des observations par année (Fig 2)}

La répartition par année est la suivante : 1 cas en 1993, 10 cas en 1994, 24 cas en 1995, 4 cas en 1996, 21 cas en 1997, 17 cas en 1998, 28 cas en 1999, 27 cas en

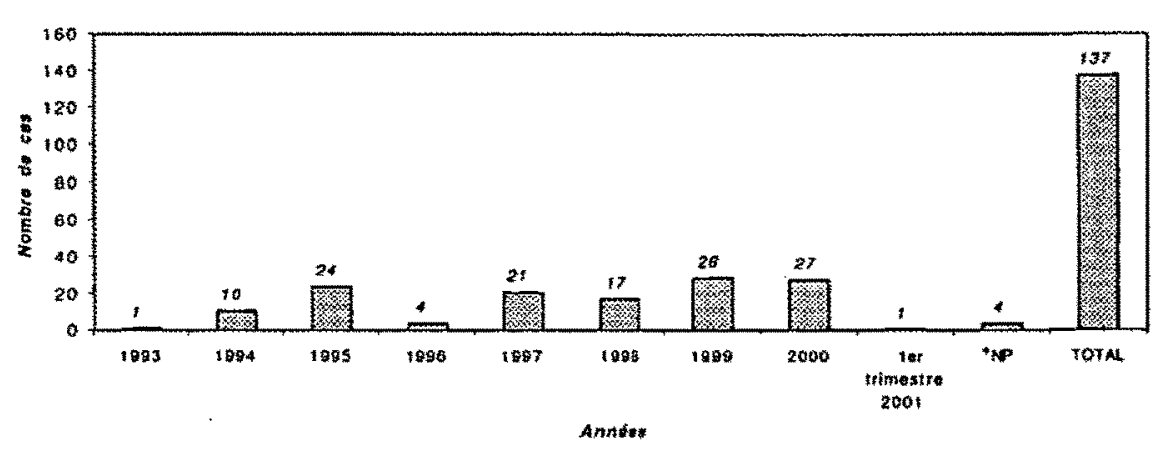

Figure 2 : Nombre de cas par année. *NP : non présisé.

2000,1 cas en 2001 (premier trimestre) ; dans 4 dossiers, la date des faits n'est pas précisée. Les observations des années 1994 et 1995 correspondent pour moitié à une série de 19 agressions commises par le même auteur (Témestaß pilé dans des aliments et ingéré à leur insu par des victimes «dépouillées» après endormissement)

\section{Profil des victimes}

Les victimes se répartissent en 65 femmes $(47,4 \%)$ et 72 hommes $(53,6 \%)$. L'âge médian est de 24 ans chez les femmes (extrêmes : 15 ans-49 ans) et de 35 ans chez les hommes (extrêmes : 25 ans-83 ans) (Fig 3).

Les âges extrêmes de la vie (enfants, vieillards) sont peu représentés.

Cent-dix (110) victimes (80\%) n'allèguent aucun traitement psychotrope ou antécédent médico-psychologique au moment des faits ; 27 victimes $(20 \%)$ allèguent un traitement par un ou plusieurs médicaments : des anxiolytiques ou hypnotiques de type benzodiazépines ou analogues (23 fois), des antidépresseurs (12 fois), des antiépileptiques [7 fois : barbituriques (4), de la carbamazépine (2), du valproate de sodium (1)], des carbamates ( 2 fois), des neuroleptiques ( 2 fois) et un traitement de substitution à l'héroïne [ 3 fois : méthadone ( 2 fois), buprénorphine haut dosage ( 1 fois)].

\section{Profil des agresseurs}

L'agresseur est un homme seul dans 78 dossiers $(57 \%)$; connu de la victime avant les faits 44 fois (relation le plus souvent récente et superficielle), inconnu de la victime 34 fois.

L'agresseur est une femme seule dans 16 dossiers $(12 \%)$, connue de la victime avant les faits 4 fois, inconnue 12 fois.

Dans 26 dossiers (18\%), la victime a été agressée par plusieurs personnes, le plus souvent plusieurs hommes, une seule fois par un couple.

Dans 19 dossiers (14\%), la victime n'a pu donner aucune information sur son agresseur.

La notion d'agresseur «spécialiste» a été retrouvée dans une série de 19 dossiers dans lesquels les victimes 
étaient endormies par l'ingestion de comprimés de Temesta ${ }^{\circledR}$ pilés et introduits dans des pâtisseries, avant d'être volées. L'agresseur contactait ses victimes, toutes des hommes homosexuels, par un réseau minitel spécialisé.

Dans 1 dossier $(0,72 \%)$, l'agresseur s'est révélé porteur du virus VIH ; la victime a bénéficié d'un traitement prophylactique anti VIH et n'a pas été contaminée.

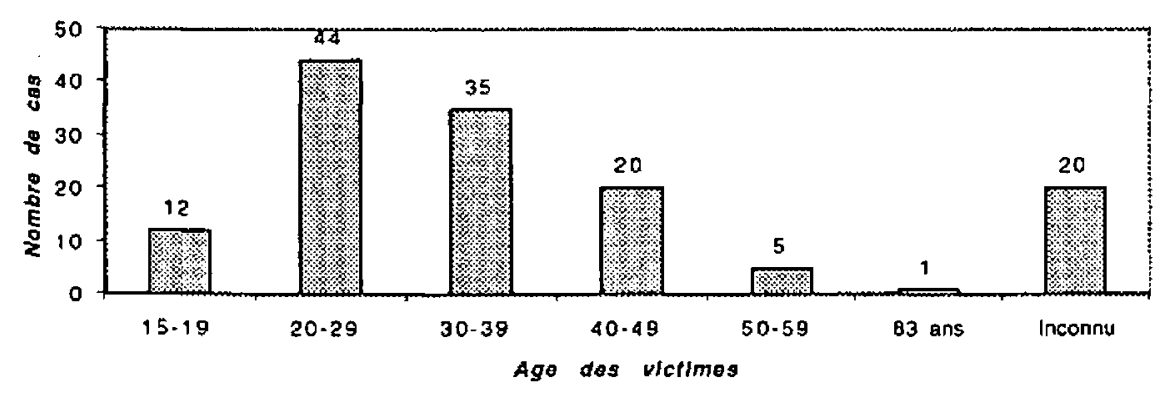

Figure 3 : Répartition des cas $(n=137: 72 H / 65 F)$ selon l'âge des victimes.

\section{Nature ou mobile de l'agression (Fig 4)}

Dans 57 dossiers (41\%), l'agression alléguée est une agression sexuelle ; la victime est une femme 49 fois, un homme 8 fois (rapports homosexuels).

Dans 62 dossiers (45\%), la victime allègue avoir été volée (chéquier, carte bleue, argent..) ; elle est de sexe masculin 60 fois.

Dans 7 dossiers $(5 \%)$ des violences physiques sont retrouvées de façon isolée.

Les autres mobiles sont des farces ( 2 dossiers) et des «neutralisations sexuelles» dans un contexte de divorce ( 2 dossiers). Aucun viol intraconjugual sous soumission n'a été répertorié.

Dans 7 dossiers ( $5 \%$ ), la nature et le mobile de l'agression sont inconnus (victime amnésique).

\section{Nature des produits supposés contenir la substance psycho-active}

La substance psycho-active a été incorporée dans une boisson non alcoolisée dans 48 dossiers ( $34 \%$ ), dans une boisson alcoolisée dans 35 dossiers $(26 \%)$, ou dans des aliments (bonbons, pâtisseries) dans 28 dossiers $(21 \%)$.

Dans 5 dossiers ( $4 \%$ ), la victime a ingéré des comprimés sous la menace ; dans 4 dossiers ( $3 \%$ ), des comprimés lui ont été proposés; dans un dossier la victime a subi une injection intramusculaire sous la contrainte. Dans 16 dossiers (12\%), la nature du produit susceptible de contenir la substance psycho-active est inconnue (victime amnésique).

\section{Symptomatologie retrouvée ou rapportée chez les victimes}

- Au moment des faits délictueux ou criminels, les symptômes les plus fréquemment rapportés sont :

- Une somnolence ou un endormissement dans 62 dossiers (46\%)

- Un tableau évoquant une «victime active mais soumise» dans 14 dossiers $(10 \%)$ : la victime participe contre sa volonté à certains actes (actes sexuels, signature de chèques, retrait d'argent) ; elle semble présenter une perte du discernement et une diminution de ses capacités de défense.

- Dans les autres dossiers (61 dossiers) sont retrouvés de façon moins systématisée : vertiges, confusion, ralentissement psychomoteur.

La durée des symptômes dépend de la demi-vie d'élimination du psychotrope incriminé (de quelques heures à 24 heures environ)

- A distance des faits (> à 24 h) :

- L'amnésie est le symptôme le pluṣ fréquemment rapporté ; elle est retrouvée dans $74 \%$ des dossiers (101 dossiers). Il s'agit d'une amnésie antérograde qui débute immédiatement après l'ingestion de la substance psycho-active ; elle est le plus souvent définitive ; la victime récupère parfois à distance quelques souvenirs partiels. L'amnésie s'accompagne constamment d'une anxiété majeure.

Des lésions traumatiques corporelles ou gynécologiques sont retrouvées dans 34 dossiers ( $25 \%$ ).

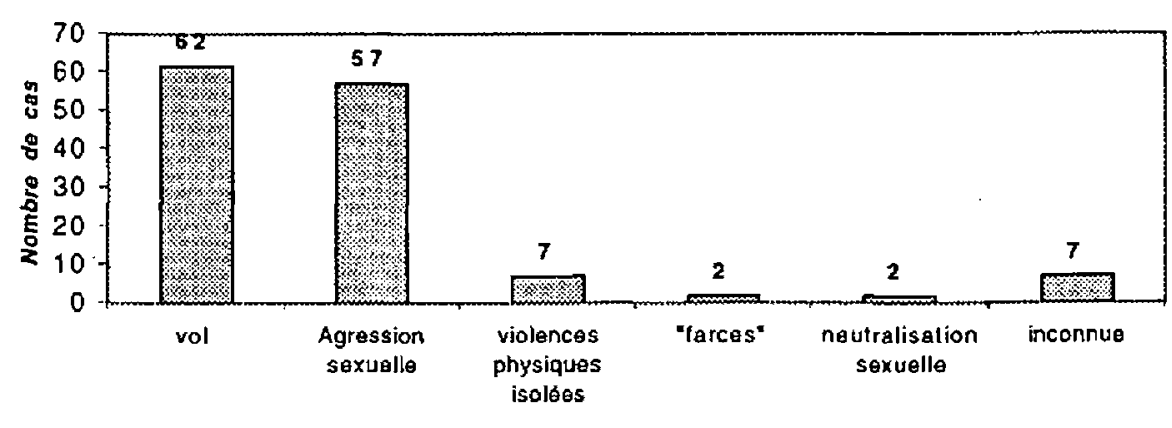

Figure 4 : Nature de l'agression $(n=137)$.

\section{Substances psycho-actives suspectées}

\section{. Aveux de l'agresseur : 17 dossiers (Fig 5)}

Dans 17 dossiers, le produit psycho-actif administré à la victime a été identifié exclusivement par des aveux de l'agresseur (analyses toxicologiques manquantes ou négatives) obtenus lors de l'enquête policière ou de l'instruction judiciaire. Dans ces dossiers, le rôle du produit psycho-actif dans la soumission est peu contestable ; l'imputabilité a donc été considérée comme forte : 


\section{- Lorazépam (Temesta®) : 15 dossiers \\ - Zolpidem (Stilnox®) : 2 dossiers}

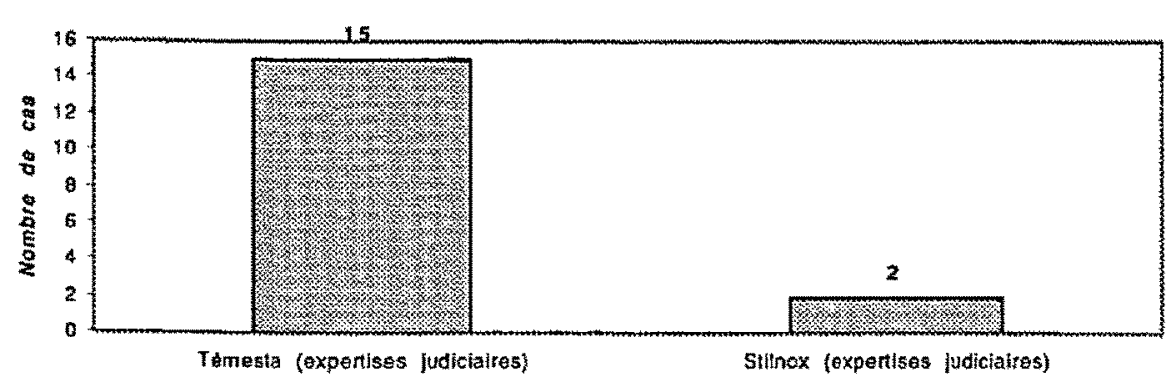

Figure 5 : Cas avec produits identifiés par des aveux ou des éléments d'interrogatoire: 17.

\section{- Analyses toxicologiques : 120 dossiers}

Les résultats des analyses toxicologiques sont disponibles dans 125 dossiers $(91 \%)$. Elles sont positives dans 120 dossiers, négatives dans 5 dossiers (dans ces 5 dossiers, les prélèvements ont toujours été pratiqués avec un délai supérieur à 3 jours après les faits). Elles ont été pratiquées sur le sang et les urines et pour un dossier, dans la boisson ingérée par la victime. L'alcool a été dosé par méthode enzymatique et chromatographique (CG/SM), les stupéfiants (opiacés, cannabis, cocaïne, amphétamines) et les autres psychotropes (notamment les médicaments) ont été recherchés par méthode immunochimique dans tous les dossiers; dans 90 dossiers sur 125 , l'immunochimie a été complétée par une technique chromatographique (CG/SM ou HPLC/ barrette de diodes).

\section{Résultats des analyses toxi- cologiques}

\section{$\left.1^{\circ}\right)$ Médicaments}

\section{Benzodiazépines et analogues : 102 dossiers}

Des benzodiazépines ou analogues de benzodiazépines (zolpidem, zopiclone) sont retrouvés par les analyses toxicologiques urinaires dans 102 dossiers : 18 fois par immunochimie seule (technique chromatographique non pratiquée), 84 fois par une double analyse immunochimique et chromatographique (CG/SM essentiellement).

Dans les 84 dossiers concernés par la double analyse, la technique chromatographique (CG/SM dans la majorité des cas ou HPLC/barrettes de diodes dans un cas) a permis l'identification des molécules dans les urines.

Les résultats sont détaillés ci-dessous $(97$ molécules identifiées pour 84 dossiers) :

Oxazépam : 26 dossiers
Aminochlorobenzophénone : 7 dossiers

Desméthyldiazépam : 7 dossiers

Bromazépam : 11 dossiers

Diazépam : 10 dossiers

Lorazépam : 8 dossiers

Flunitrazépam : 7 dossiers

Flurazépam : 3 dossiers

Tétrazépam : 3 dossiers

Oxazépam + acéprométazine : 3 dossiers

Nitrazépam : 3 dossiers

Clobazam : 2 dossiers

Alprazolam : 2 dossiers

Clonazépam : 2 dossiers

Lormétazépam : 1 dossier

Zolpidem : 1 dossier

Zopiclone : 1 dossier (en HPLC/barrettes de diodes)

Remarques:

- Le nombre total de molécules identifiées (97) est supérieur au nombre de dossiers (84) : plusieurs benzodiazépines ont pu être administrées à la même victime et un seul médicament peut-être à l'origine de plusieurs métabolites urinaires (exemple: Valium ${ }^{\circledR}=>$ oxazépam, desmethyldiazépam, diazépam).

- Oxazépam, desméthyldiazépam, aminochlorobenzophénone sont des métabolites communs à plusieurs benzodiazépines:

. Oxazépam + aminochlorobenzophénone : métabolites urinaires communs à Librax $($, Lysanxia(®), Noctran ${ }^{\circledR}$, Seresta ${ }^{\circledR}$, Tranxène ${ }^{\circledR}$,

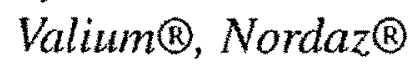

- Desméthyldiazépam : métabolite urinaire commun à Lysanxia ${ }^{\circledR}$, Tranxène ${ }^{\circledR}$, Valium ${ }^{\circledR}$

La chromatographie a permis de positiver 4 prélèvements urinaires négatifs en immunochimie : clobazam (1), clonazépam (1), zolpidem (1) par CG/SM et zopiclone (1) par HPLC/barrettes de diodes (il n'existe pas de test immunochimique commercialisé pour ces deux dernières molécules)

L'imputabilité des benzodiazépines ou analogues détectés a été considérée comme forte dans 73 dossiers sur 102.

Dans 23 dossiers, la victime alléguait un traitement par benzodiazépines ou analogues, le plus souvent retrouvé dans les urines. Dans 6 dossiers, bien que non signalée, la consommation habituelle de benzodiazépines par la victime pouvait être suspectée au regard des antécédents et du terrain (toxicomane, pathologie psychiatrique). Dans ces 29 dossiers où les circonstances de l'agression étaient peu claires (allégations discordantes, alcoolisation associée), l'imputabilité des benzodiazépines ou analogues a été considérée comme douteuse. 


\section{Antihistaminiques sédatifs : 12 dossiers}

Des anti-histaminiques sédatifs (pour lesquels il n'existe pas de tests immunochimiques commercialisés) ont été détectés au cours des analyses toxicologiques par chromatographie gazeuse couplée à la spectrométrie de masse dans 12 dossiers :

Doxylamine : 5 dossiers

Hydroxyzine : 5 dossiers

Buclizine : 2 dossiers

Forte imputabilité dans 6 dossiers sur 12

\section{Neuroleptiques : 7 dossiers}

Des neuroleptiques ont été identifiés par les analyses toxicologiques en CG/SM dans 7 dossiers :

Cyamémazine : 6 dossiers (dans 2 dossiers, la cyamémazine faisait partie du traitement habituel de la victime)

Loxapine : 1 dossier (injection intramusculaire sous la contrainte)

Forte imputabilité dans 3 dossiers sur 7 [cyamémazine (2), loxapine (1)].

\section{Barbituriques : 5 dossiers}

Du phénobarbital a été identifié par les analyses toxicologiques en double analyse (immunochimie et CG/SM) dans 5 dossiers; dans 2 de ces dossiers, le phénobarbital faisait partie du traitement habituel de la victime.

Forte imputabilité dans 3 dossiers sur 5

\section{Trihexyphénidyle (Artane ${ }^{\circledR}$ ) : 2 dossiers}

Identifié par CG/SM dans 2 dossiers (forte imputabilité).

\section{Tramadol : I dossier}

Identifié par CG/SM dans 1 dossier (forte imputabilité).

D'autres médicaments ont été identifiés au cours des analyses toxicologiques mais leur responsabilité dans la soumission est peu probable; il s'agit en effet de traitements en cours ou de traitements probables compte-tenu des antécédents allégués :

. Toloxatone (Humorilß) : 1 dossier

. Clomipramine (Anafranil囚) : 1 dossier

Méprobamate : 3 dossiers (traitement en cours par Equanil ${ }^{\circledR}$ dans 2 dossiers)

. Citalopram : 3 dossiers (traitement en cours).

Les techniques chromatographiques (CG/SM) ont permis de positiver 17 prélèvements urinaires dont les analyses toxicologiques s'étaient révélées négatives en immunochimie (Tableau I) :

doxylamine (3 dossiers), hydroxyzine (3 dossiers), GHB (1 dossier), buclizine ( 2 dossiers), trihexyphénidyle (Artane $\left.{ }^{\circledR}\right)$ ( 2 dossiers), zolpidem (1 dossier), zopiclone (1 dossier), tramadol (1 dossier), clobazam (1 dossier), clonazépam (1 dossier), LSD (1 dossier).
Dans un autre dossier, l'analyse de la boisson a permis la détection de GHB.

Tableau I : Molécules détectées dans les urines par chromatographie gazeuse couplée à la spectrométrie de masse (CG/SM) et passées inaperçue en immunochimie (17 dossiers).

\begin{tabular}{|c|l|}
\hline Doxylamine & 3 dossiers \\
Hydroxyzine & 3 dossiers \\
Buclizine & 2 dossiers \\
Trihexyphénidyle & 2 dossiers \\
Zolpidem & 1 dossier \\
Zopiclone & 1 dossier (par HPLC/Barettes de diodes) \\
Tramadol & 1 dossier \\
Clobazam & 1 dossier \\
Clonazépam & 1 dossier \\
GHB & 1 dossier \\
LSD & 1 dossier \\
\hline
\end{tabular}

Commentaires: En dehors du clobazam et du clonazépam (benzodiazépines), il n'existe pas de tests immunochimiques commercialisés pour les autres molécules.

\section{$2^{\circ}$ ) Substances non médicamenteuses}

\section{GHB (gamma hydroxybutyrate de sodium) : 2} dossiers

(Remarque : le gamma hydroxybutyrate de sodium est également un médicament anesthésique (gamma $\mathrm{OH}$ ), inscrit sur liste I et réservé à l'usage hospitalier. Le médicament semble peu concerné par le mésusage qui concerne surtout des préparations clandestines et «artisanales»).

Du GHB a été retrouvé en CG/SM dans deux dossiers :

- Dans la boisson ingérée par la victime : 1 dossier (analyse pratiquée par le laboratoire Toxlab - Paris)

- Dans les prélèvements sanguins d'une victime pratiqués une dizaine d'heures après les faits à des taux faibles mais supra-physiologiques $(7,5 \mathrm{mg} / \mathrm{l}): 1$ dossier

L'imputabilité est forte dans les deux dossiers (symptomatologie évocatrice, agression sexuelle documentée par une recherche de spermatozoïdes positive, taux biologiques supra-physiologiques)

\section{Alcool : retrouvé dans 47 dossiers}

Dans tous ces dossiers, l'alcool a été consommé de façon volontaire. Son rôle potentialisateur avec les autres psychotropes identifiés est probable.

\section{Stupéfiants :}

Cannabis dans 29 dossiers

Opiacés dans 10 dossiers 


\section{Cocaïne dans 7 dossiers}

Amphétamines dans 4 dossiers

LSD dans 1 dossier (forte imputabilité) (analyse pratiquée par le laboratoire Toxlab - Paris)

En dehors du cas où a été retrouvé du LSD (mis dans du café, ingéré par la victime à son insu et identifié dans des urines prélevées 5 heures après les faits), les autres stupéfiants ont été consommés de façon volontaire.

Leur rôle dans la soumission est difficile à apprécier compte-tenu des informations disponibles. Leur détection dans les urines semble être le reflet d'une consommation habituelle et antérieure aux faits.

\section{Imputabilité du produit psycho-actif identi- fié}

L'imputabilité dans le phénomène de soumission de la substance psycho-active identifiée est considérée comme forte dans 108 dossiers sur 137 (78 \%). Les produits les plus suspects sont rapportés figure 6 .

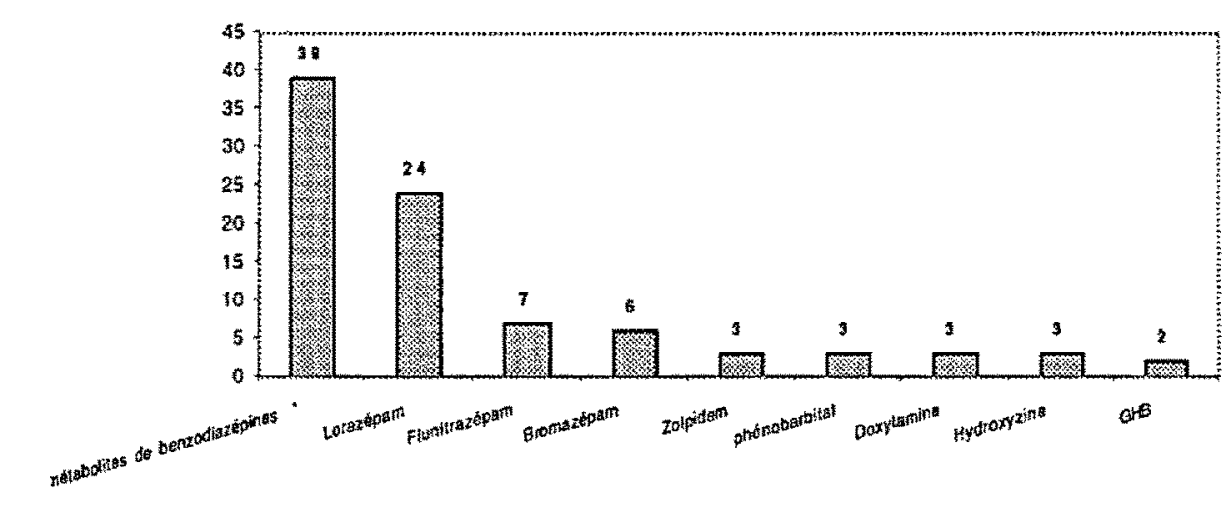

Figure 6: Produits impliqués les plus suspects (imputabilité forte : 108 dossiers sur 137) (Identification par analyses toxicologiques ou aveux).

Autres : clonazépam (2), cyamémazine (2), diazépam (2), flurazépam (2), Oxazepam-acéprométazine (Noctran) (2), tétrazépam (2), trihexyphénidyle (2), loxapine (1), zopiclone (I), tramadol (1), LSD (1).

* Les métabolites communs à plusieurs benzodiazépines ont été identifiés sans que l'on puisse remonter avec certitude à la molécule mère.

\section{Discussion}

\section{Nombre de cas}

Sur la période concernée (1993-2001), le nombre de cas est sensiblement stable depuis 1997.

Ces chiffres ne refiètent pas la réalité du phénomène dont la sous évaluation est évidente. Afin de quantifier la soumission chimique, d'identifier les substances utilisées et d'améliorer le prise en charge des victimes, il est souhaitable de mettre en place des réseaux de collecte et d'analyse des cas impliquant les cliniciens, les Centres Anti-Poisons (CAP), les Centres Régionaux de
Pharmaco-Vigilance (CRPV), les Centres d'Évaluation et d'Information sur la Pharmacodépendance (CEIP), sur l'ensemble du territoire national.

\section{Caractéristiques des victimes et nature de l'agression}

Les femmes sont très majoritairement victimes d'agression sexuelle ( 49 femmes pour 8 hommes); les hommes sont très majoritairement dépouillés de leur argent ( 60 hommes pour 2 femmes).

Les âges extrêmes de la vie (vieillards, enfants) sont peu représentés : seulement une victime a moins de 16 ans (une jeune fille de 15 ans) et une plus de 75 ans (un homme de 83 ans); cette faible proportion est peut-être liée au fait que ces victimes, plus dépendantes que les autres, accèdent plus difficilement aux soins et aux démarches judiciaires (dépôt de plainte).

Quatre-vingt pour cent $(80 \%)$ des victimes n'allèguent aucun traitement psychotrope au moment des faits ; dans la grande majorité des dossiers de l'étude, la mise en cause d'un tel traitement dans la symptomatologie observée et dans la positivité des recherches toxicologiques peut ainsi être exclue.

Les lésions traumatiques associées sont peu fréquentes ( $25 \%$ des dossiers) ; cela peut témoigner des difficultés qu'éprouve la victime à résister physiquement à l'agresseur, sous l'effet du produit psycho-actif (effet sédatif, hypnotique ou myorelaxant).

\section{Caractéristiques des agresseurs}

L'agresseur est majoritairement un homme seul (78 dossiers) ; il est le plus souvent inconnu de la victime ou connu d'elle depuis peu. Dans notre étude, l'agresseur fait rarement parti de ses proches ; il n'a pas été retrouvé de cas dans le cadre de violences conjugales mais il est probable que des cas de soumission existent également entre sujets proches et intimes. La notion d'agresseur «spécialiste» n'a été retrouvée qu'une seule fois (série de 19 dossiers avec utilisation de Temesta ${ }^{\circledR}$ pilé dans des pâtisseries); on peut penser, que cette technique d'agression est parfois réutilisée plusieurs fois par un même auteur, même si cette hypothèse n'est pas soutenue par les résultats de cette étude. L'observation du phénomène doit être affinée pour préciser ces points.

Dix-neuf victimes $(14 \%)$ ne peuvent donner la moindre information sur l'agresseur présumé à cause d'une amnésie profonde ou des circonstances de certaines agressions qui surviennent au cours de rassemblements festifs favorisant l'anonymat (substance versée dans un verre provisoirement abandonné par son propriétaire). 


\section{Symptomatologie rapportée chez la vic- time (1),(6),(7),}

$\mathrm{Au}$ moment des faits, la victime présente un endormissement ou des troubles de conscience autorisant vols et abus sexuels ; plus rarement, la victime est «active», consciente mais soumise, commettant des actes contre sa volonté ( signature de chèques, utilisation de cartes bancaires, participation à des actes sexuels) : ces deux tableaux peuvent se succéder en fonction des stimuli extérieurs. Ils précèdent une amnésie d'installation progressive dans des délais qui dépendent de la demi-vie d'élimination du produit ; cette amnésie est antérograde (débute au moment de l'ingestion du produit psycho-actif), fixée et le plus souvent définitive ; très anxiogène, l'amnésie peut-être à l'origine d'explications fantaisistes ou confuses pouvant être prises pour des affabulations et nuisant à la crédibilité de la victime au cours de son parcours judiciaire (dépôt de plainte) ou médical. Ce tableau clinique relativement caractéristique doit être connu des professionnels concernés (policiers, médecins) afin d'optimiser la prise en charge des victimes : envoi rapide vers des urgences hospitalières pour prélèvements à visée toxicologique et conservatoire et acheminement de ceux-ci vers des laboratoires possédant l'équipement analytique adapté ; l'administration de médicaments anxiolytiques ou psycho-actifs avant la réalisation de ces prélèvements doit être proscrite.

\section{Prélèvements biologiques et analyses toxicologiques (7),(8),}

Les prélèvements biologiques (sang et urines) doivent être pratiqués le plus tôt possible. Dans notre expérience, la probabilité de détecter un médicament psychoactif administré à une victime à son insu est très faible au delà de 48 heures. Des prélèvements positifs au delà de cette limite ne sont retrouvés que dans 4 dossiers de notre série : bromazépam (Lexomil@) $(55 \mathrm{~h})$, hydroxyzine $(\operatorname{Atarax} \circledast)(56 \mathrm{~h})$, flurazépam (non commercialisé en France) (72 h) et lorazépam (Temesta $₫) ~(72 ~ h)$. Pour le GHB, le délai est beaucoup plus court (une dizaine d'heures) (9).

Les victimes doivent donc être médicalisées rapidement afin de favoriser des prélèvements précoces dont la réalisation nécessite le consentement de l'intéressé et qui doivent être acheminés vers des laboratoires possédant l'équipement nécessaire. Des prélèvements conservatoires (sang et urines) doivent être congelés $\left(-20^{\circ}\right)$, à l'abri de la lumière (surtout si du LSD est suspecté) et conservés pour les besoins d'une éventuelle enquête médico-judiciaire ultérieure. Des prélèvements de cheveux peuvent être utile en cas de délais dépassés (8) ; leur exploitation est le plus souvent ordonnée par un magistrat dans le cadre d'une enquête policière (flagrance) ou d'une instruction ; elle est difficilement envisageable en routine actuellement.

Les analyses toxicologiques doivent être constamment effectuées sur le sang et les urines et nécessitent des techniques chromatographiques [(chromatographie en phase gazeuse couplée à la spectrométrie de masse (CG/SM), chromatographie liquide à haute pression couplée à un détecteur à barrettes de diodes (HPLC/barrettes)] en complément des techniques immunochimiques, de sensibilité et de spécificité insuffisantes ; ces dernières techniques utilisées en routine dans un contexte de surdosage, ne sont pas adaptées aux faibles posologies et aux faibles taux biologiques fréquemment retrouvés dans les cas de soumission; de plus, elles ne détectent pas certaines familles médicamenteuses, comme les analogues de benzodiazépines (zolpidem, zopiclone), les antihistaminiques sédatifs, le trihexyphénidyle (Artane(B) ou d'autres psychotropes comme le GHB, la kétamine et le LSD. Dans notre série, dix-sept prélèvements urinaires négatifs en immunochimie ont pu être «positivés» grâce aux techniques chromatographiques; ce résultat concerne des molécules indétectables en immunochimie [(antihitaminiques sédatifs ( 8 dossiers), analogues de benzodiazépines ( 2 dossiers) , GHB (1 dossier), tramadol (1 dossier), trihexyphénidyle (Artane®) (2 dossiers) et LSD ( 1 dossier)] et des benzodiazépines (clobazam et clonazépam) passées inaperçues avec les techniques immunochimiques insuffisamment sensibles (2 dossiers),

En matière de soumission chimique, doivent être systématiquement recherchés les médicaments habituellement incriminés (benzodiazépines et analogues, antihistaminiques sédatifs, neuroleptiques, anticholinergiques) mais aussi alcool, cannabis, cocaïne, opiacés, LSD, GHB, kétamine. D'autres recherches peuvent être orientées par des éléments complémentaires recueillis en clinique ou dans le cadre de l'enquête judiciaire.

\section{Substances psycho-actives incriminées}

Elles sont incorporées majoritairement dans des boissons ( 82 dossiers), le plus souvent non alcoolisées (48 dossiers) ; la présence d'alcool n'est donc pas nécessaire pour obtenir l'effet recherché. Plus rarement, l'incorporation se fait dans des aliments. Aucune victime ne signale avoir constaté un goût ou une coloration particulière au moment de l'ingestion. Afin de prévenir ou limiter leur utilisation à des fins de soumission, des modifications de galénique des médicaments psychotropes pourraient être plus fréquemment proposées par les laboratoires pharmaceutiques ou imposées par les 
pouvoirs publics. Cela a été fait pour le comprimé de Rohypnolß $1 \mathrm{mg}$ depuis Juin 1998 : ajouté à un liquide, il se délite en plus de 15 minutes, laisse un dépôt à la surface et libère une coloration bleue qui modifie la couleur d'origine de la boisson.

\section{$\left.1^{\circ}\right)$ Médicaments}

Des benzodiazépines et analogues sont incriminés dans 119 dossiers $(87 \%$ ) (dans 17 dossiers grâce aux aveux de l'agresseur, dans 102 dossiers grâce aux analyses toxicologiques) ;

leur imputabilité est forte dans 90 dossiers (aveux de l'agresseur, pas de traitement psychotrope allégué par la victime) ; pratiquement toutes les benzodiazépines ou analogues sont représentés en particulier lorazépam (23 dossiers), bromazépam (11 dossiers), diazépam (10 dossiers), flunitrazépam ( 7 dossiers), zolpidem ( 3 dossiers). Dans $40 \%$ des dossiers environ les recherches urinaires ont mis en évidence des métabolites communs à plusieurs benzodiazépines (oxazépam, desmethyldiazépam, benzophénone), ne permettant pas une identification plus précise de la molécule-mère ; l'identification sanguine complémentaire paraît ainsi intéressante, notamment dans un contexte judiciaire surtout si les prélèvements sanguins sont précoces.

Parmi les autres médicaments psychotropes incriminés, les antihistaminiques sédatifs arrivent par ordre de fréquence en deuxième position ( 12 dossiers, soit $9 \%$ ), nettement après les benzodiazépines ; parmi eux, la doxylamine ( 3 dossiers) et l'hydroxyzine ( 3 dossiers) sont retrouvées avec une forte imputabilité. Leur utilisation dans la soumission a déjà été rapportée dans la littérature (10); dans notre série, l'effet sédatif domine, à l'origine d'un endormissement des victimes autorisant vol ou abus sexuel.

Par ordre de fréquence, viennent ensuite les neuroleptiques dans 7 dossiers dont 3 avec une forte imputabilité [cyamémazine ( 2 fois) et loxapine ( 1 fois)], le phénobarbital dans 5 dossiers dont 3 avec un forte imputabilité, le trihexyphénidyle (Artane(B) dans 2 dossiers avec une forte imputabilité et le tramadol dans 1 dossier avec une forte imputabilité. Les symptômes rapportés par les victimes sont sédation, confusion, apathie, vertiges et amnésie.

\section{$\left.2^{\circ}\right)$ Substances non médicamenteuses}

En ce qui concerne les substances non médicamenteuses, la prise d'alcool est retrouvée (par l'interrogatoire et les analyses toxicologiques) dans 47 dossiers (34\%). L'alcool a toujours été consommé de façon volontaire et ne peut-être rendu responsable à lui seul de la soumission telle que nous l'avons définie (consommation par la victime à son insu); néanmoins, son effet favorisant est peu discutable, lorsqu'il est associé à un autre psychotrope notamment un médicament ; la prise d'alcool est absente dans deux-tiers des dossiers et n'est donc pas nécessaire à la soumission.

Le GHB n'a été retrouvé que dans deux dossiers de la série : une fois dans la boisson ingérée par la victime, une autre fois dans le sang de la victime à des taux supra-physiologiques $(7,5 \mathrm{mg} / \mathrm{l})$ dans des prélèvements pratiqués 10 heures environ après les faits. Dans les deux cas la victime a été vue précocément à l'hôpital (dans les dix premières heures); dans un cas, la victime, confuse et tenant des propos à caractère sexuel, a été conduite à l'hôpital par un proche, témoin de son comportement inhabituel et inexplicable selon lui par une simple prise d'alcool ; dans l'autre cas la victime, partiellement amnésique, craignant d'avoir été sexuellement abusée au cours d'une soirée, s'est présentée spontanément et rapidement à l'hôpital ; dans les deux cas, la symptomatologie associait un syndrome confusionnel de quelques heures précédant une amnésie plus ou moins totale (quelques «flashs»). Ce tableau doit faire évoquer le diagnostic et pratiquer au moindre doute, dans ce contexte, un examen gynécologique et des prélèvements locaux ; dans ces deux cas, l'agression sexuelle a été documentée par une recherche de spermatozoïdes positive au niveau vaginal.

La mise en évidence du GHB dans les liquides biologiques est difficile compte-tenu de sa demi-vie d'élimination courte (30 à 60 minutes) (9) ; les prélèvements biologiques et la prise en charge médicale doivent donc être très précoces ; l'intervention de l'entourage peut être capitale en conduisant la victime rapidement en milieu hospitalier. Les prélèvements doivent être acheminés vers des laboratoires équipés [chromatographie gazeuse couplée à la spectrométrie de masse (CG/SM) indispensable] (7). L'interprétation des résultats toxicologiques est compliquée par une production endogène de GHB dont les taux physiologiques sont inférieurs à $2 \mathrm{mg} / \mathrm{l}$ dans le sang et à $10 \mathrm{mg} / \mathrm{l}$ dans les urines (11). Une recherche au niveau des cheveux est possible mais de pratique difficile et non réalisable en routine (11). Finalement, les cas documentés d'agression sexuelle après administration de GHB à l'insu d'une victime sont encore très peu nombreux ; sa diffusion réelle est difficile à apprécier compte-tenu des difficultés liées à sa détection et à l'interprétation des analyses toxicologiques (8), (9),(11).

En dehors du cas avec identification de LSD dont l'imputabilité est forte, le rôle des autres stupéfiants (cannabis, cocaïne, opiacés) est plus difficile à établir ; ils ont été consommés volontairement par la victime et leur détection semble être plutôt le reflet d'une consommation antérieure aux faits ; dans certaines observations, l'effet sédatif favorisant du cannabis et 
des opiacés avec d'autres psychotropes comme l'alcool et des médicaments est probable.

\section{Conclusion}

Les benzodiazépines et leurs analogues (zolpidem, zopiclone) sont les substances les plus fréquemment utilisées dans la soumission chimique du fait de leurs propriétés amnésiantes, sédatives et désinhibitrices. Pratiquement toutes les benzodiazépines et analogues de benzodiazépines sont représentés. Les autres médicaments ou substances incriminées sont plus rarement les anti-histaminiques sédatifs, les neuroleptiques et plus récemment le GHB dont la diffusion réelle est mal appréciée. L'association à l'alcool n'est pas nécessaire à la soumission. Le rôle des stupéfiants et en particulier du cannabis, est difficile à apprécier et mérite d'être mieux étudié.

La détection des produits psycho-actifs dans les liquides biologiques n'est possible que si les victimes sont rapidement prises en charge ; cela suppose une sensibilisation des victimes elles-mêmes, mais aussi de l'entourage et des professionnels concernés (gérants d'établissements à vocation festive, policiers, médecins) ; une information du grand public et de ces professionnels est donc souhaitable.

Les prélèvements biologiques doivent bénéficier de techniques analytiques chromatographiques (CG/SM), indispensables dans ce contexte ; les laboratoires équipés doivent être identifiés.

La soumission chimique est un acte délictueux ou criminel. Tout doit être mis en ouvre pour ne pas compromettre une éventuelle judiciarisation ultérieure. Il est d'ailleurs souhaitable d'inciter les victimes à déposer plainte rapidement auprès des autorités judiciaires afin qu'elles bénéficient sans retard des circuits de prise en charge les plus adaptés incluant notamment les urgences ou consultations médico-judiciaires mises en place sur l'ensemble du territoire national.

Le recensement et l'analyse des cas doivent se poursuivre afin d'évaluer l'ampleur du phénomène et de suivre au plus près l'évolutivité des produits utilisés ; comme en matière de toxicomanie et de pharmacodépendance, ceux-ci sont susceptibles de changer et l'émergence de nouvelles molécules est également à craindre dans ce domaine.

\section{Références}

1. Bismuth C, Dally S. Soumission médicamenteuse. In : Cas cliniques en toxicologie. Paris : Flammarion, 1994 : 82-3.

2. Bismuth C,Dally S, Borron SW. Chemical submission/ GHB, benzodiazepines and other knock out drops. J Toxicol Clin Toxicol $1997 ; 35$ : 595-8.

3. Questel F, Becour B, Diamant Berger O. Drogués à leur insu : une prise en charge souvent déficiente. A propos de quelques observations exemplaires. J Med Leg Droit Med $1999 ; 42: 77-80$.

4. Stark MM, Wells D. Dug-mediated sexual assault. J Clin Forensic Med $1999 ; 6$ : 53-5.

5. ZecchiniL. La drogue américaine du "cambriolage sexuel parfait". Le Monde, $1^{\text {er }}$ Octobre 1996: 1.

6. Questel F, Becour B, Dupeyron JP, Galliot-Guilley M, Diamant-Berger $O$. Soumission médicamenteuse : 4 ans d'expérience aux UMJ de Paris. J Med Leg Droit Med $2000 ; 43: 459-465$.

7. Lagier G, Questel F, Elkharrat D, Diamant-Berger O. Prise en charge des victimes droguées à leur insu. Med Leg Hosp 1999 ; 2 : 113-14.

8. Kintz P. La soumission chimique. Aspects analytiques et expertises médico-légales. Ann Toxicol. Anal. 2001 ; $13: 120-1$.

9. Ghysel MH. Le GHB : l'acide Gamma Hydroxy Butyrique. Revue de la littérature, Toxicorama, 1999 ; $10: 1-11$.

10. Vasseur P. Agression sexuelle après soumission médicamenteuse. Med Leg Hosp. $2000 ; 3: 94$.

11.Gaillard Y, Goulle JP, Pépin G. Le GHB dans le sang, les urines et les cheveux : interprétation des résultats. $9^{\text {ime }}$ congrès de la Sociétế Fraçaise de Toxicologie Analytique. La Clusaz : 12-16 Mars 2001. 